\title{
A NEW PULSATIONAL RADIUS FOR DELTA CEPHEI
}

\author{
DAVID G. TURNER \\ Department of Astronomy, Saint Mary's University, Halifax, Nova Scotia B3H 3C3, Canada \\ Received 24 May 1988; revised 5 August 1988
}

\begin{abstract}
The Baade-Wesselink method has been reapplied to existing photometric and radial-velocity measures for the classical Cepheid $\delta$ Cephei, but a different approach to the analysis was adopted in which spectrophotometric narrowband $K H G$ observations were used to isolate phase pairs of identical atmospheric temperature. Corrections for variable spectral-line blocking and changing effective gravity in the observed $V$ magnitudes and $B-V$ colors were analyzed semiempirically, and a solution obtained by minimizing the relative error in the result. The mean radius found for $\delta$ Cephei is $42.7 R_{\odot} \pm 1.0 R_{\odot}$, which is quite close to other recently derived radius estimates for this Cepheid, including theoretical values obtained from the secondary bump on its light curve.
\end{abstract}

\section{INTRODUCTION}

The derivation of pulsational radii for Cepheids and RR Lyrae variables has become a popular area of research in recent years. The analytical techniques used have generally been developed from a scheme proposed years ago by Baade (1926) and later developed for practical use by Wesselink (1946), namely the Baade-Wesselink method. Although several variations of the general method have appeared ( see Gautschy 1987), the basic principle still consists of identifying phase pairs of identical surface brightness for a pulsating star. Such phase pairs invariably correspond to points of unequal brightness on the star's light curve, so magnitude differences between them can be numerically converted into ratios of the star's radii at these phases. Integration of the star's observed radial-velocity variations, suitably adjusted for projection effects to the star's actual radial excursions, yields the actual differences in radii between these phases as well as the differences of each from the star's mean radius. The mean radius itself is therefore easy to calculate, usually from an average of the results for several phase pairs or from an appropriately weighted mean of the values from the whole cycle.

In Wesselink's (1946) original study of $\delta$ Cephei, phases of identical surface brightness were identified with phases of identical color index. This fundamental assumption that surface brightness is a single-valued function of color for pulsating stars seems to be a matter deserving of further study. Evans (1976b) has noted that it is possible to test the assumption by plotting radius ratios versus radius differences for the various phase pairs. Enough information exists on Cepheid radius variations to predict that, when plotted for phases running from light maximum to light minimum, the data should trace out a closed loop which approximates a straight line passing through the point $(0,1)$. The slope of this line should be close to the reciprocal of the minimum radius (Abt 1959). The loop (if one could properly refer to it as such) should be traced out in a clockwise sense owing to the fact that the smaller of each radius pair lies closer to mean radius at light minimum than at light maximum, while the "upper" series of points are produced near the initial decline from light maximum when the smaller of each radius pair is near minimum radius (each data point connects to $(0,1)$ with slope $1 /\left(R_{\min }+\Delta\right)$, where $\Delta$ is the deviation of the smaller radius from $\left.R_{\text {min }}\right)$. In fact, an open loop traced out in a counterclockwise sense is what is typically observed (Evans 1976b, 1980). Correction of the observed colors for varying contributions of microturbulence during the pulsational cycle seems to improve the situation considerably (Evans 1980; Benz and Mayor 1982). However, this step relies upon transformations based upon model atmospheres or upon results from "normal" stars, and may not be strictly applicable to pulsating variables.

This paper presents a new determination of the mean radius of $\delta$ Cephei using existing observational data and a modified version of the standard Baade-Wesselink type of analysis. The primary difference with respect to previous analyses lies in the use of a narrowband spectral-line photometric index to isolate phase pairs of identical surface brightness. The $K H G$ index established by observers at Brigham Young University (McNamara and Potter 1969; McNamara et al. 1970; Feltz 1972) has been used for this purpose since it has been found to be an excellent index of effective temperature for Cepheids (Turner et al. 1987). A semiempirical approach has been adopted in analyzing the data in order to take into account the phase-dependent microturbulence and effective gravity variations (see Evans (1980); Benz and Mayor (1982)). This method of analysis has been found to produce results with quite small internal errors. The findings amply demonstrate the power of the method, and also yield a mean radius for $\delta$ Cephei that appears from various tests to be a very close approximation to its true mean radius.

\section{METHOD OF ANALYSIS}

The method of analysis followed here has many similarities to those used in other treatments, although no new observations of $\delta$ Cephei were obtained. Published observational data were used exclusively, and proper phasing of these was effected using the well-defined long-term record of the Cepheid's period variations described and summarized by Szabados (1980). This step should minimize any problems arising from incorrect phase matching of the photometric and radial-velocity observations (Fernie and Hube 1967).

The photoelectric $B V$ data were taken from Mitchell et al. (1964), Evans (1976a), Feltz and McNamara (1980, transformed to the Johnson system using the relations of Cousins (1987)), Szabados (1980), and Moffett and Barnes (1980), while the radial-velocity measures were those of Shane (1958), Evans (1976a), and Barnes et al. (1987). No differences in velocity phasing, amplitude, or zero point were apparent between the older photographic velocities and the more recent radial-velocity spectrometer measures, al- 
though a small amplitude difference might have been expected according to the study by Hindsley and Bell (1986). The measuring errors in Shane's velocities are smaller than those of Barnes et al., so a small amplitude effect in the latter velocities could easily go undetected. Most previous determinations of the pulsational radius of $\delta$ Cephei have relied upon the velocity curve delineated by Shane's observations, which have excellent phase coverage in addition to excellent quality. The present study likewise relies quite heavily upon Shane's radial-velocity observations.

$K H G$ data for this study were taken from Feltz and McNamara (1980). The $K H G$ index is defined by narrowband interference filters centered on $\mathrm{Ca}$ II $\lambda 3933, \mathrm{H} \delta \lambda 4101$, and $\mathrm{CH} \lambda$ 4305, respectively, and like the $\mathrm{H} \beta$ index is an excellent diagnostic of temperature for Cepheid variables (Turner et al. 1987). The present analysis depends critically upon the $K H G$ observations, so special care was taken to accurately identify the phase dependence of the index. Due to the sparse nature of the $K H G$ dataset, Feltz and McNamara's (1980) $\mathrm{H} \beta$ observations of $\delta$ Cephei were also included. The latter data were found to be linearly related to the $K H G$ observations, and helped to reduce the noise level in the $K H G$ data when averaged with them. Running phase means were formed with all available observations (see Fig. 1), and smooth curves drawn through these values were used in the subsequent pulsational radius analysis.

For illustrative purposes, a classical Baade-Wesselink analysis was performed using the $B V$ data of Fig. 1. Phase pairing was made using steps of $0.03 \mathrm{mag}$ in $B-V$ color, and displacement values $D_{1}$ and $D_{2}$ (uncorrected for projection effects) were taken from an integration of the radial-velocity curve. The results of this analysis are plotted in Fig. 2, and illustrate the problems typical of the classical approach (Evans 1976b). The points define a moderately open loop, somewhat more open than expected, which is traced out in a counterclockwise rather than clockwise sense. After elimination

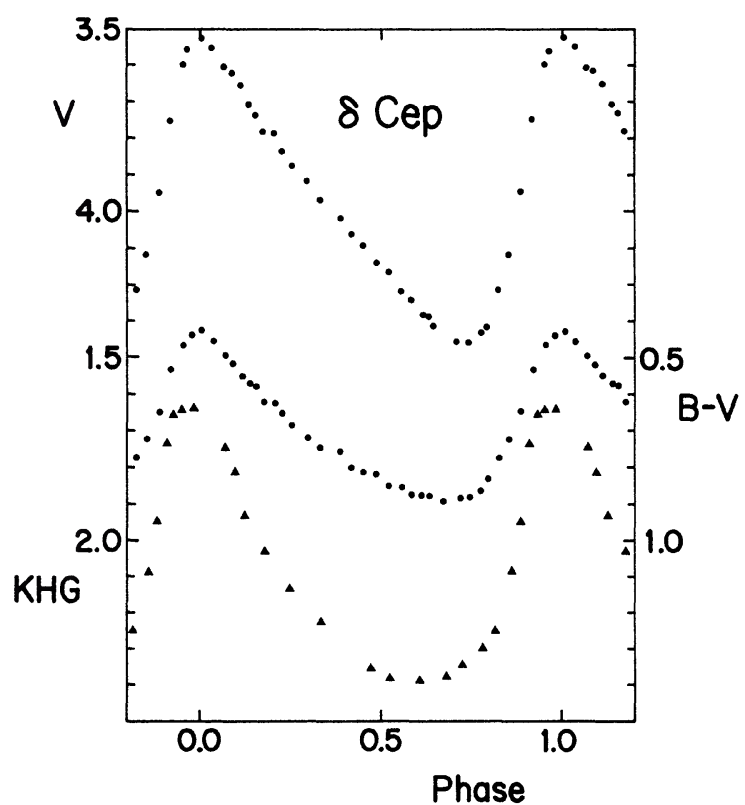

FIG. 1. Phase-averaged brightness and color variations of $\delta$ Cephei derived from published data. The $K H G$ values incorporate $\mathrm{H} \beta$ photometry transformed to the same system.

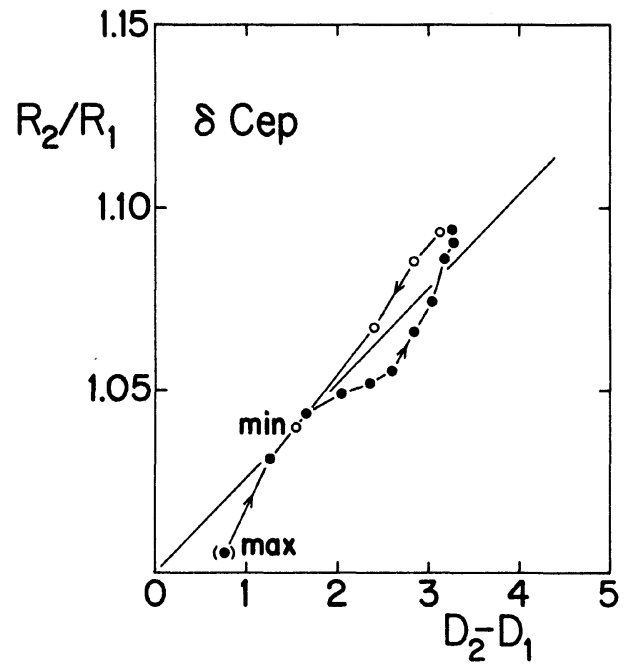

FIG. 2. Classical Baade-Wesselink solution for the radius of $\delta$ Cephei using phases of identical $B-V$, with displacement differences $D_{2}-D_{1}$ uncorrected for projection effects. Expansion-cycle points (dark circles) are distinguished from contraction-cycle points (light circles), and the fitted line corresponds to a radius of $52.8 R_{\odot}$.

of the anomalous point near light maximum, an average over the other data values yields an uncorrected radius for $\delta \mathrm{Ce}$ phei of $40.3 R_{\odot} \pm 1.2 R_{\odot}$ s.e. $(2.96 \%$ relative error). The value of the projection factor in this case (photographic velocities at a dispersion of $11 \AA \mathrm{mm}^{-1}$ ) is $p=1.31 \pm 0.03$ (Parsons 1972; Karp 1975b). The resulting pulsational radius is $52.8 R_{\odot} \pm 2.6 R_{\odot}$, a value on the high side of the range from 31 to $60 R_{\odot}$ (mean $45.3 R_{\odot} \pm 1.6 R_{\odot}$ ) derived in previous classical Baade-Wesselink analyses of $\delta$ Cephei (Fernie 1984).

It may be evident from the data of Fig. 1 that phases of identical $K H G$ index do not correspond to phases of identical $B-V$ color. The observed color differences for $\delta$ Cephei range from $\sim 0.04$ to $\sim 0.08$ in $B-V$. One reason for this lies in the phase-dependent microturbulence variations of $\delta$ Cephei and other Cepheids (see Evans 1980; Benz and Mayor 1982), which can be understood in terms of a phase dependence of the velocity gradient in their atmospheres (Karp 1973). The effect is to produce phase-dependent linewidth variations (in addition to those arising from projection effects) which alter the amount of line blocking in the stellar continuum in a systematic sense. As a greater number of spectral lines lie in the blue continuum band than in the visual continuum band, $B$ magnitudes are affected more than $V$ magnitudes, and color differences in $B-V$ are expected. A similar effect can also be produced by variations in the effective surface gravity of Cepheids during their cycles (cf. Karp 1975a, Pel 1978). This complicates the pulsational analysis since any color differences $\Delta(B-V)$ arising from variations in line broadening or gravity must be tied to corresponding magnitude shifts, which we designate here by the simple relation $\Delta V=K \Delta(B-V)$. Such corrections must be subtracted from the observed magnitude differences to allow proper radius ratios to be calculated between phases of matching $K H G$.

The $K H G$ spectral-line index is itself subject to effects arising from microturbulence and gravity variations, but appar- 
ently to a lesser degree than the $B-V$ index. Feltz (1972), for example, was unable to detect a surface-gravity dependence of $K H G$ in his study of B, A, and $\mathrm{F}$ type dwarfs and giants, while Turner et al. (1987) were unable to detect a significant surface gravity or microturbulence dependence of $K H G$ for their calibrating Cepheids. The loop opening for $\delta$ Cephei evident in a plot of $K H G$ versus $B-V$ (or $b-y$ ) can apparently be explained by the effects of variable microturbulence on the continuum index (see Turner et al. 1987). $K H G$ can be quite noticeably affected by the presence of companions to Cepheids, but for single Cepheids it seems to be a more ideal temperature index than $B-V$ (or any other continuum index).

Simple line blocking (as compared to line blanketing) should produce a positive value of $K$ according to the arguments presented earlier. This is confirmed by an inspection of the data of Fig. 1. Negative values of $K$ (as might arise from backwarming) serve only to generate unrealistic radius estimates for $\delta$ Cephei. Published model atmospheres rarely take into account line-blocking effects on the continuum due to microturbulence differences. The model atmospheres of Kurucz (1979), for example, incorporate line absorption into the derived continua values, but not variations in line blocking, as would arise from variations in microturbulence. From an inspection of the various Kurucz stellar atmosphere predictions of brightness and color for stars with temperatures and gravities typical of Cepheids, we estimated that pure line-blocking effects on Cepheid continua should be described by values of $K$ lying between 0 and 3 . Consequently, $K$ was left as a free parameter lying in this range to be solved for in a semiempirical type of analysis. It appears to have some similarities to the color term $b$ employed by Ivanov (1984) in his inverted version of the Baade-Wesselink method. Upon consideration of arguments presented by Scarfe (1976) and Evans (1976b), we decided that minimization of the percentage error in the radius would probably generate an optimum value for the parameter $K$ required in the solution.

An initial solution for $K$ is illustrated in Fig. 3. Phase pairing was made with the data of Fig. 1 using steps of 0.05 mag in $K H G$, and the initial solution incorporated data from

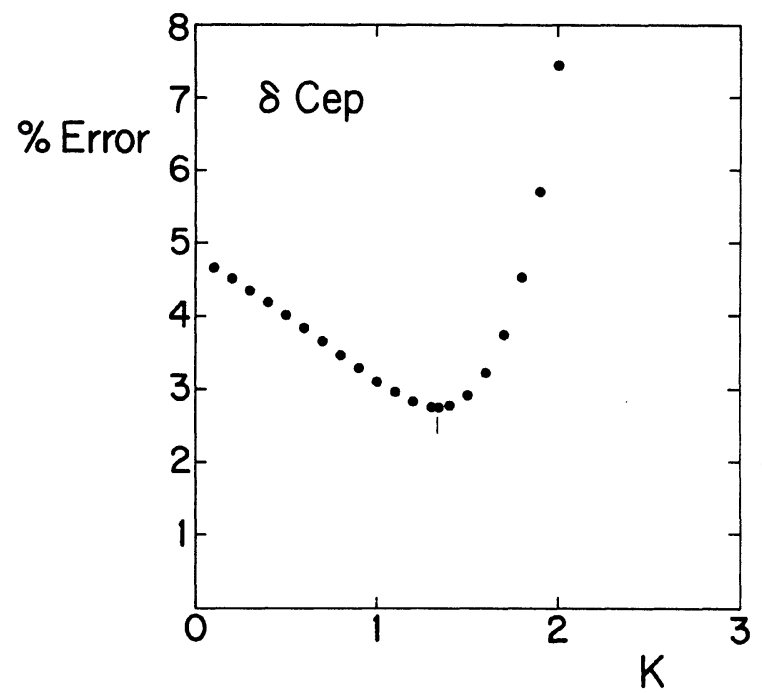

FIG. 3. Solution for the line-blocking factor $K$ by minimization of the relative error in radius. Minimum occurs at $K=1.335$. the entire pulsational cycle. The smallest relative error in radius $(2.75 \%)$ occurs for $K=1.335$, and a general solution over all phase pairs which makes use of this value is illustrated in Fig. 4. The uncorrected radius in this case is $30.4 R_{\odot} \pm 0.8 R_{\odot}$ s.e., which becomes $39.8 R_{\odot} \pm 1.4 R_{\odot}$ after adjustment for projection effects.

Although the relative error in this solution is slightly smaller than in the classical case, it is evident from Fig. 4 that the data exhibit the same type of problem as illustrated in Fig. 2, namely a moderately open loop traced out in the reverse sense from that expected. That a dichotomy exists in $K$ is demonstrated by the displacement of points (light circles) in Fig. 4 arising from phase pairs on the contraction cycle of $\delta$ Cephei. All other phase pairs (dark circles) arise from at least one point on the expansion cycle of the Cepheid. Such a dichotomy also appears to exist in the data of Fig. 2.

The ultimate cause of this dichotomy appears to originate with the variations in effective gravity of $\delta$ Cephei during its cycle. Its existence implies that separate solutions for $K$ must be obtained for the different sets of phase pairs. Figure 5 illustrates the effect of solving separately for $K$ using phase pairs from the expansion and contraction cycles of the Cepheid. Expansion-cycle points yield $K=1.36$ and an uncorrected radius of $32.5 R_{\odot} \pm 0.3 R_{\odot}$ (relative error $0.87 \%$ ), while contraction-cycle points yield $K=1.89$ and an uncorrected radius of $32.8 R_{\odot} \pm 0.3 R_{\odot}$ (relative error $0.92 \%$ ). Finer subdivision of the data does not appear to be warranted. Note that both radius estimates agree within their error despite markedly different solutions for $K$. A final solution, illustrated in Fig. 6, is based upon a combination of all of the data. The result is an uncorrected radius of $32.6 R_{\odot} \pm 0.2 R_{\odot}$ (relative error $0.68 \%$ ), which becomes $42.7 R_{\odot} \pm 1.0 R_{\odot}$ when adjusted for projection effects. The tightness and clockwise sense of delineation for the "loop" in Fig. 6 are precisely the characteristics expected in a valid Baade-Wesselink analysis.

\section{DISCUSSION}

Changes in effective gravity during Cepheid pulsational cycles affect their colors in addition to line-blocking varia-

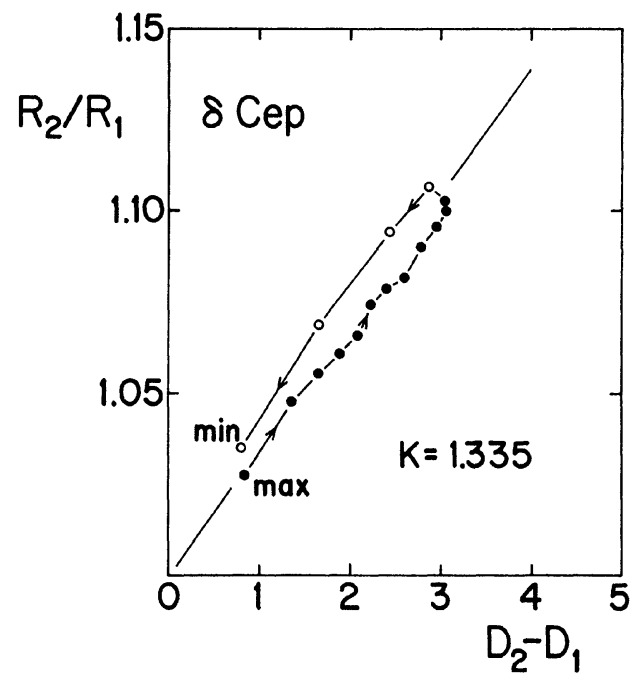

FIG. 4. Baade-Wesselink solution for $\delta$ Cephei using phases of identical $K H G$, with symbols as in Fig. 2. The fitted line corresponds to a radius of $39.8 R_{\odot}$. 


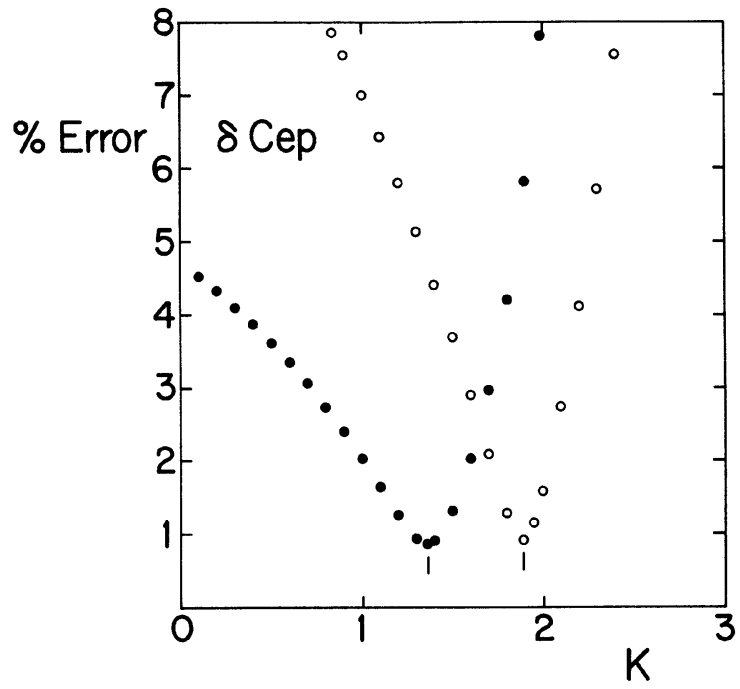

Fig. 5. Separate solutions for the line-blocking factor $K$ for expansioncycle points (dark circles, minimum at $K=1.36$ ) and contractioncycle points (light circles, minimum at $K=1.89$ ).

tions, and to first order our separation of phase pairs from the expansion cycle relative to those from the contraction cycle takes such gravity changes into account quite effectively. Expansion-cycle phase pairs correspond to slightly higher effective gravities than do contraction-cycle phase pairs (Karp 1975a; see also Evans 1980). The model atmospheres of Kurucz (1979) indicate that increasing the gravity for giants and supergiants with effective temperatures similar to those of classical Cepheids tends to produce a negative shift of the $K$ factor defined here. This qualitatively matches the separation of $K$ values seen in Fig. 5, although explicit modeling of this effect (and variable line blocking) would be quite useful. The generation of virtually identical radius estimates from quite distinct solutions for $K$ using separate groups of phase pairs seems to be a reasonably effective test of the validity of this type of approach. The test works ex-

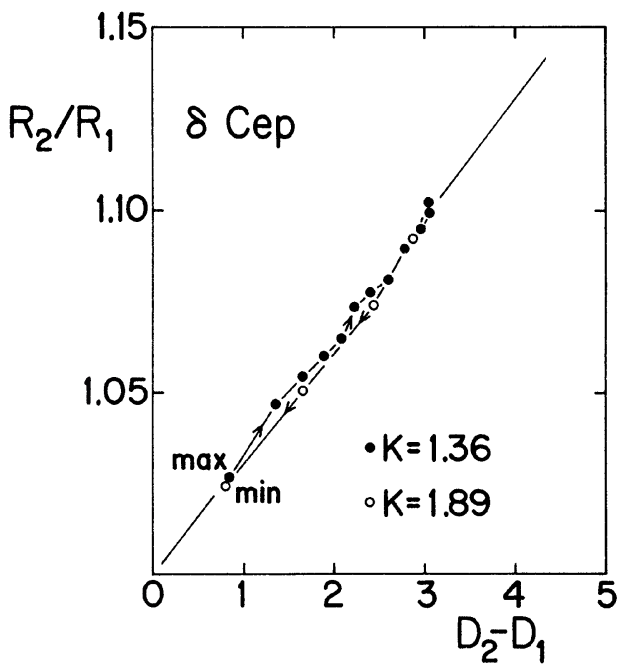

FIG. 6. Final Baade-Wesselink solution for $\delta$ Cephei using phases of identical $K H G$, with symbols as in Figs. 2, 4, and 5. The fitted line corresponds to a radius of $42.7 R_{\odot}$. tremely well for $\delta$ Cephei, although the excellent quality of the observational data for this star makes it somewhat of a special case.

Additional tests with other Cepheids are in progress, and it appears that the constancy of $K$ for phase pairs on the contraction cycle may not be true for all Cepheids. However, $K$ does seem to be constant during expansion-cycle phase pairs, and varies slightly from one Cepheid to another in a manner consistent with the decrease of mean gravity with increasing pulsational period.

Table I summarizes the results of the present study, and compares the newly derived radius estimate for $\delta$ Cephei with other recent determinations taken from the literature, with theoretical predictions based upon the secondary bump at phase 0.7 in its light curve (Carson and Stothers 1988), and with the photometrically derived radius for a cluster Cepheid (CV Mon) of nearly identical period. The most recent application of the surface-brightness technique by Moffett and Barnes (1987) and the inverted Baade-Wesselink method of Ivanov (1984) yield radius estimates for $\delta$ Cephei that agree remarkably well with the value obtained here. It is not clear why techniques that assume that surface brightness is related to color should give valid pulsational radii, although it may relate to the manner in which $V$ and $B-V($ or $V-R$ ) vary during Cepheid cycles. The use of color indices in the red part of the spectrum may reduce the problem, since continuum magnitudes in the infrared should be less affected by microturbulence and gravity variations. However, nearly all magnitude estimates would appear to be affected to some degree, so the problem of obtaining valid radius ratios remains.

Predictions for the "bump" radius of $\delta$ Cephei obtained from nonlinear pulsation models depend upon the model opacities used, as described by Carson and Stothers (1988). The radius determined for $\delta$ Cephei in this paper agrees almost exactly with the bump radius predicted using the Carson opacities, and perhaps provides some support for the continued use of Carson opacities in such models. In any case, it is significant that there is excellent agreement in this instance between observational and theoretical results (see Carson and Stothers 1988). The values obtained in Table I provide excellent support for the radius estimate obtained in this paper, despite the very different assumptions upon which the derivations of these values are based.

Conversion of the present radius estimate for $\delta$ Cephei into an estimate of its luminosity requires information on its

TABLE I. Radius estimates for $\delta$ Cephei.

\begin{tabular}{|c|c|c|}
\hline $\begin{array}{l}\text { Radius } \\
\left(R_{\odot}\right)\end{array}$ & Method & Source \\
\hline $45.3 \pm 1.6$ & $\begin{array}{l}\text { Classical B-W methods } \\
\text { (summary) }\end{array}$ & Fernie (1984) \\
\hline $40 \pm 3$ & Inverted B-W method & Ivanov (1984) \\
\hline $41.4 \pm 2.4$ & Surface-brightness method & Moffett and Barnes (1987) \\
\hline $42.7 \pm 1.0$ & $K H G$-based B-W method & This paper \\
\hline 37.2 & $\begin{array}{l}\text { Secondary bump (C-S } \\
\text { opacities) }\end{array}$ & Carson and Stothers (1988) \\
\hline 42.4 & $\begin{array}{l}\text { Secondary bump (Carson } \\
\text { opacities) }\end{array}$ & Carson and Stothers (1988) \\
\hline $43.0 \pm 3.0$ & Cluster fitting for CV Mon & Turner (1978); this paper \\
\hline
\end{tabular}


mean effective temperature. This step is somewhat uncertain since it requires information on the space reddening of the Cepheid and the adoption of an appropriate effective-temperature scale (see Pel 1985). With the space reddening found for $\delta$ Cep by Turner et al. (1987), the unreddened mean color of the Cepheid is $(\langle B\rangle-\langle V\rangle)_{0}=0.57$. This corresponds to $\left\langle T_{\text {eff }}\right\rangle=5874 \pm 26 \mathrm{~K}$ (our estimated uncertainty) using the recently derived temperature scale of Teays and Schmidt (1987). With $T_{\odot}=5770 \mathrm{~K}$ and $M_{\text {bol }_{\odot}}=4.75$, we obtain $\left\langle M_{\text {bol }}\right\rangle=-3.48 \pm 0.06$ and $\left\langle M_{V}\right\rangle=-3.45$ \pm 0.06 for $\delta$ Cephei. Nearly identical values would be predicted for the Cepheid from its pulsational period according to recently published calibrations of the Cepheid periodluminosity (PL) relation (for example, the relations of Caldwell and Coulson (1987) yield $\left\langle M_{V}\right\rangle=-3.24$ and $\left\langle M_{V}\right\rangle=-3.33$ from their PL and PLC relations, respectively).

An independent check on the present radius estimate is possible using the cluster Cepheid calibration of the PL relation. Published calibrations tend to incorporate the results of cluster studies of varying quality, often with slightly different assumptions about reddening corrections and the zero point of the main-sequence calibration. The present comparison has therefore been restricted to one specific object, namely CV Mon, a cluster Cepheid with a period that differs from that of $\delta$ Cephei by only $+0.24 \%$.

Photometrically derived distances and reddenings for the CV Mon cluster have been published by Turner (1978) and Schmidt (1983). The results of both studies are in reasonably good agreement, but we have chosen to adopt the former for reasons described elsewhere (Turner 1986). The intrinsic color and luminosity for CV Mon are therefore $(\langle B\rangle-\langle V\rangle)_{0}=0.61$ and $\left\langle M_{V}\right\rangle=-3.35 \pm 0.05$. An uncertainty of \pm 0.15 is probably more realistic for the absolute magnitude, given the uncertainty in the Hyades distance modulus, which is $3.2 \pm 0.1$ for this particular comparison (see Turner 1986). Adoption of these values with the Teays and Schmidt (1987) temperature scale, a bolometric correction of -0.05 , and the largest likely uncertainties in bolometric magnitude and mean temperature results in an estimated radius of $43.0 R_{\odot} \pm 3.0 R_{\odot}$ for CV Mon. A number of new studies of the CV Mon cluster are currently in progress, but it seems unlikely that they will significantly alter the excellent agreement between the derived photometric radius of CV Mon and the pulsational radius of $\delta$ Cephei found here. It seems reasonable to conclude that $\mathrm{CV}$ Mon and $\delta$ Cephei are of virtually identical dimension to well within the uncertainties of the estimates.

All of the tests that can be applied to the present estimate for the mean radius of $\delta$ Cephei confirm its general validity within the context of currently available information on the dimensions of classical Cepheids. The method by which this radius was obtained appears to be quite powerful, and very closely satisfies the basic conditions of the Baade-Wesselink technique. The internal error of the result obtained here is quite small (less than 1\%). In fact, the relative uncertainty of the correction factor for the radial velocities is larger than the internal uncertainty in radius, and ultimately dominates the quoted uncertainty in the final estimate. However, the small size of the internal errors may be partly illusory since they quite likely relate to the excellent quality of the observational data for $\delta$ Cephei. Tests are currently in progress to extend the technique to a larger sample of Cepheids, where observational uncertainties are much more important.

It is a pleasure to thank Jim Burke for organizing the observational data and doing much of the groundwork for this study. Financial support from the Natural Sciences and Engineering Reseach Council of Canada, in the form of a University Research Fellowship and Individual Operating Grant to D.G.T. and a University Undergraduate Student Research Award to Jim Burke, is gratefully acknowledged.
Abt, H. A. (1959). Astrophys. J. 130, 824.

Baade, W. (1926). Astron. Nachr. 228, 359.

Barnes, T. G., Moffett, T. J., and Slovak, M. H. (1987). Astrophys. J.

Suppl. 65, 307

Benz, W., and Mayor, M. (1982). Astron. Astrophys. 111, 224.

Caldwell, J. A. R., and Coulson, I. M. (1987). Astron. J. 93, 1090.

Carson, T. R., and Stothers, R. B. (1988). Astrophys. J. 328, 196.

Cousins, A. W. J. (1987). Observatory 107, 80.

Evans, N. R. (1976a). Astrophys. J. Suppl. 32, 399.

Evans, N. R. (1976b). Astrophys. J. 209, 135.

Evans, N. R. (1980). In Current Problems in Stellar Pulsation Instabilities, edited by D. Fischel, J. R. Lesh, and W. M. Sparks, NASA Tech. Mem. No. 80625 (NASA, Washington, DC), p. 237.

Feltz, K. A., Jr. (1972). Publ. Astron. Soc. Pac. 84, 497.

Feltz, K. A., Jr., and McNamara, D. H. (1980). Publ. Astron. Soc. Pac. 92, 609.

Fernie, J. D. (1984). Astrophys. J. 282, 641.

Fernie, J. D., and Hube, J. O. (1967). Publ. Astron. Soc. Pac. 79, 95.

Gautschy, A. (1987). Vistas Astron. 30, 197.

Hindsley, R., and Bell, R. A. (1986). Publ. Astron. Soc. Pac. 98, 881.

Ivanov, G. R. (1984). Astrophys. Space Sci. 105, 369.

Karp, A. H. (1973). Astrophys. J. 180, 895.

Karp, A. H. (1975a). Astrophys. J. 200, 354.

Karp, A. H. (1975b). Astrophys. J. 201, 641.

\section{REFERENCES}

Kurucz, R. L. (1979). Astrophys. J. Suppl. 40, 1.

McNamara, D. H., Helm, T. M., and Wilcken, S. K. (1970). Publ. Astron. Soc. Pac. 82, 293.

McNamara, D. H., and Potter, D. (1969). Publ. Astron. Soc. Pac. 81, 545.

Mitchell, R. I., Iriarte, B., Steinmetz, D., and Johnson, H. L. (1964). Bol.

Obs. Tonantzintla Tacubaya 3, 153.

Moffett, T. J., and Barnes, T. G. (1980). Astrophys. J. Suppl. 44, 427.

Moffett, T. J., and Barnes, T. G. (1987). Astrophys. J. 323, 280.

Parsons, S. B. (1972). Astrophys. J. 174, 57.

Pel, J. W. (1978). Astron. Astrophys. 62, 75.

Pel, J. W. (1985). In Cepheids: Theory and Observations, IAU Colloquium

No. 82, edited by B. F. Madore (Cambridge University, Cambridge), p. 1.

Scarfe, C. (1976). Astrophys. J. 209, 141.

Schmidt, E. G. (1983). Astron. J. 88, 104.

Shane, W. W. (1958). Astrophys. J. 127, 573.

Szabados, L. (1980). Mitt. Sternw. Ungarischen Akad. Wiss. Budapest No. 76.

Teays, T. J., and Schmidt, E. G. (1987). Lect. Not. Phys. 274, 173.

Turner, D. G. (1978). J. R. Astron. Soc. Can. 72, 248.

Turner, D. G. (1986). Astron. J. 92, 111.

Turner, D. G., Leonard, P. J. T., and English, D. A. (1987). Astron. J. 93, 368.

Wesselink, A. J. (1946). Bull. Astron. Inst. Neth. 10, 91. 